IDENTIFICATION OF SCAPULAR KINEMATICS USING SURFACE

MAPPING: A VALIDATION STUDY

by

Jeffrey Mattson

A thesis submitted to the Faculty of the University of Delaware in partial fulfillment of the requirements for the degree of Master of Science in Biomechanics and Movement Science

Fall 2011

Copyright 2011 Jeffrey Mattson

All Rights Reserved 


\title{
IDENTIFICATION OF SCAPULAR KINEMATICS USING SURFACE MAPPING: A VALIDATION STUDY
}

\author{
by \\ Jeffrey Mattson
}

Approved:

James Richards, Ph.D.

Professor in charge of thesis on behalf of the Advisory Committee

Approved:

William Farquhar, Ph.D.

Chair of the Department of Kinesiology and Applied Physiology

Approved:

Kathleen Matt, Ph.D.

Dean of the College of Health Sciences

Approved:

Charles G. Riordan, Ph.D.

Vice Provost for Graduate and Professional Education 


\section{ACKNOWLEDGMENTS}

James Richards, Ph.D., for inspiring me to stay in academia by demonstrating how fun research can be and for helping to expand my knowledge and skill-set necessary to satisfy my curiosity.

William Rose, Ph.D., and Jill Higginson, Ph.D., for being a part of my committee and providing valuable and thoughtful input.

Steph Russo, for her mutual interest in a quest to better understand scapular kinematics.

My family and friends, for their unconditional love and support. 


\section{TABLE OF CONTENTS}

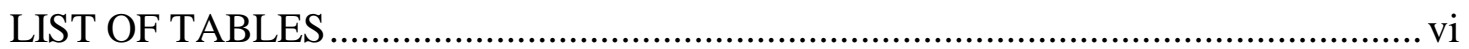

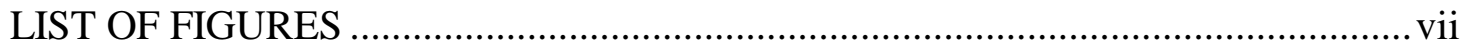

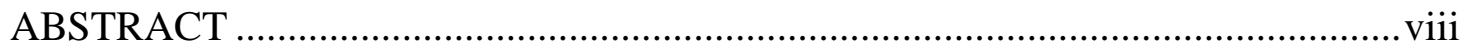

Chapter

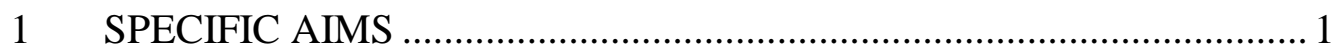

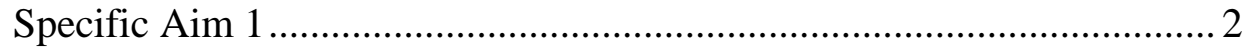

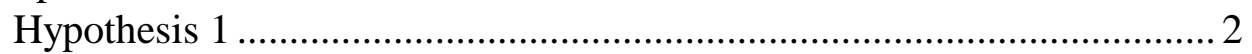

Specific Aim 2 2............................................................................. 2

Hypothesis 2 ..................................................................................... 3

2 BACKGROUND AND SIGNIFICANCE .............................................. 4

3 METHODS

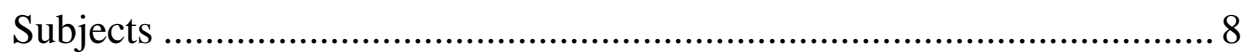

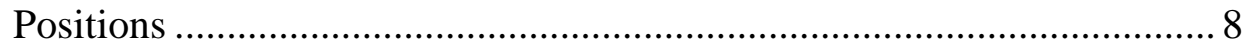

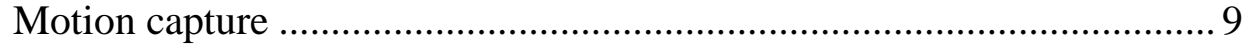

Spherical fit trial ......................................................................... 9

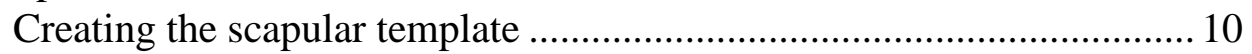

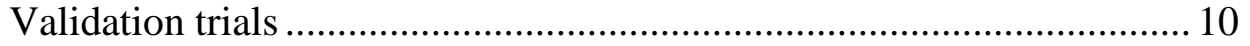

Grid surface map trials ...................................................................... 11

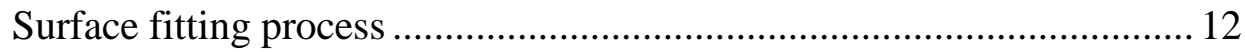

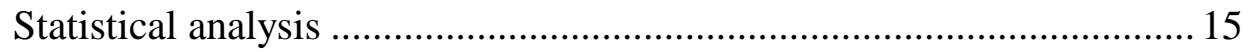

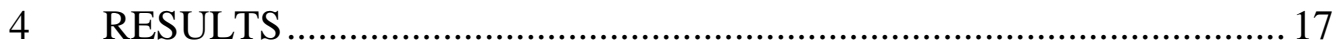

Sensitivity of filtering, grid density, and cluster size …........................... 17

Validation of surface mapping …………............................................... 19

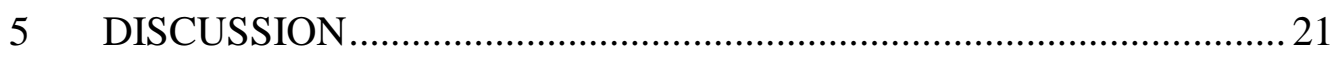

Comparison to existing methods ............................................................ 21 


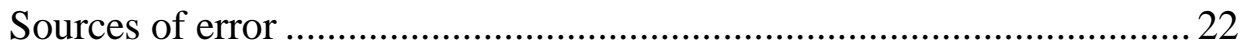

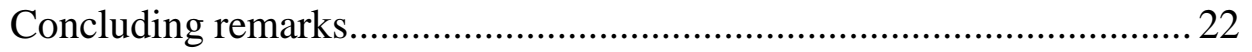

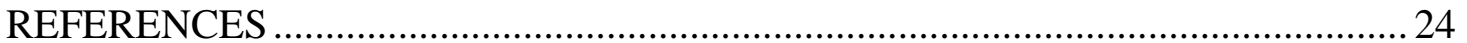

Appendix

A ADDITIONAL COMMENTS ............................................................ 29

B IRB APPROVAL LETTER.............................................................. 31 


\section{LIST OF TABLES}

Table 1 Subjects first held their arm in a neutral position to create a scapular template, then the positions of the modified Mallet classification, which are clinically relevant for assessing shoulder function.

Table 2 Retroreflective, $6 \mathrm{~mm}$ spherical markers were placed on ISG recommended landmarks for the duration of data collection.

*virtually reconstructed....

Table 3 Surface mapping results using raw data and planes determined by the six nearest surface markers. Mean errors and RMS errors represent the difference in helical angles between methods of scapular and humeral orientations in the thorax coordinate system. Five trials were excluded because resultant humerus error was greater than $15^{\circ}$. Significant differences in mean error were determined by repeated measures ANOVA for each anatomical axis of each position, with method (palpation and surface map) as an independent variable. 


\section{LIST OF FIGURES}

Figure 1 Validation trial of the hand behind back position with superimposed scapular and humeral orientations for demonstration purposes.

Figure 2 Surface map of the hand behind back position with superimposed scapular and humeral orientations for demonstration purposes.

Figure 3 Visual representation of the absolute distance from one scapular landmark to its nearest plane on the grid surface formed by applying singular value decomposition.

Figure $4 \quad$ a) Graphical display of the surface fitting process. The global minimum error was found among 16,000 orientations tested in a $20^{\circ} \times 40^{\circ} \times 20^{\circ}$ range. In this case, the sum of the absolute distances between the five scapular template markers and the surface was $6.3 \mathrm{~mm}$ at the best-fit orientation and was found at the 9,496th iteration, indicated by the circle. b) Posterior view of the surface fitting result for the hand behind back position. The scapular and humeral orientations during the validation trial are shown in red. The corresponding best-fit scapular orientation found by the surface fitting process, as well as the humeral orientation and grid during the surface map trial, are shown in green.

Figure 5 Effect of surface fitting conditions on the goodness of fit. The sphere diameters indicate mean RMS errors for the resultant helical angles directly between scapulae of the validation and grid surface map trials. RMS errors were calculated across all subjects for each position, and the position RMS errors were averaged. Surface fitting conditions tested were 0th (raw), 3rd, and 5th order polynomials (red, blue, and green respectively); 15, 20, 25 and 30 interpolated markers per row (does not apply to raw data); 4, 5 and 6 nearest markers for plane determination. The smallest diameter sphere, corresponding to the best surface fitting conditions, was obtained with the raw marker data with planes based on the 6 nearest points. 18 


\begin{abstract}
The immediate goal of this study was to develop and validate a noninvasive, computational surface mapping approach for measuring scapular kinematics by using available motion capture technology in an innovative manner. The long-term goal is to facilitate the development of individualized surgical treatment plans and rehabilitation by determining the role of the scapula in children with brachial plexus birth palsy (BPBP) and other shoulder pathologies. The population for this study consisted of fourteen healthy adults with prominent scapulae; equivalent or better results are expected with BPBP patients, due to minimal soft tissue covering their scapulae. Subject-specific scapular templates were created using the coordinates of five scapular landmarks obtained from palpation with subjects seated and arms relaxed in a neutral position. The scapular landmarks were re-palpated and their locations recorded in the six arm positions of the modified Mallet classification. The six Mallet positions were repeated to create surface maps from a grid of approximately 300 markers covering the scapula. The scapular template created in the neutral position was iteratively fit to the surface map of each grid trial, providing an estimate of the orientation of the scapula. These estimates of scapular orientation were compared to the known scapular orientation determined from the scapular landmarks palpated in each Mallet position. The magnitude of the largest mean difference about an anatomical axis between the two measures of scapular orientation was $3.8^{\circ}$ with an RMS error of $5.9^{\circ}$, which is an improvement upon existing non-invasive methods of measuring scapular kinematics.
\end{abstract}




\section{Chapter 1}

\section{SPECIFIC AIMS}

The immediate goal of this thesis was to develop a clinically applicable approach to measuring scapular kinematics in individuals with minimal soft tissue overlying the scapula. The long-term goal of this research is to improve treatment planning strategies for patients with brachial plexus birth palsy (BPBP) and other impairments of shoulder function. Standardized assessments such as the modified Mallet classification are frequently used to measure the degree of shoulder dysfunction (Bae et al., 2003), but these tests provide no insight into the scapula's contribution. This is important, as treatment outcomes can be highly dependent on scapular function (Kozin et al., 2010; Nath et al., 2004; Vastamaki, 1987). However, current methods for measuring scapular kinematics have limitations (Kozin et al., 2010; Graichen et al., 2000; Bourne et al., 2009; Gomes et al., 2010; Karduna et al., 2001; Illyes and Kiss, 2006; McClure et al., 2001; van Andel et al., 2009; Bey et al., 2006; Meskers et al., 2007). The development of a precise, safe, and accessible clinical tool for the assessment of pre- and post-operative scapular function would have a positive impact on therapeutic and surgical interventions.

The approach in this study was to develop a clinical tool that can provide valid and reliable measures of scapular kinematics utilizing standard passive-marker motion capture technology applied in an innovative manner. Traditional motion 
capture involves placing three or more markers on a rigid segment to track the kinematics of the segment. This approach fails when measuring scapular kinematics as the scapula moves freely under the skin surface (Bourne et al., 2009; Gomes et al., 2010; Karduna et al., 2001; Illyes and Kiss, 2006; McClure et al., 2001; van Andel et al., 2009; Bey et al., 2006; Meskers et al., 2007). Instead, the method described below took advantage of the overlying soft tissue surface deformation as the scapula changes position.

There were two specific aims associated with this approach:

\section{$\underline{\text { Specific Aim } 1}$}

Ensure that subjects achieve matching arm positions between methods. Comparing a direct measure of scapular orientation with an estimated measure of scapular orientation required the subjects to achieve matching arm positions for both measures.

\section{Hypothesis 1}

Subjects will be recorded in arm positions that are significantly correlated with and not significantly different between the two measures for each Mallet posture.

\section{$\underline{\text { Specific Aim } 2}$}

Develop and validate a computational algorithm to identify scapular orientation by fitting a scapular template to the surface formed by the soft-tissue overlying the scapula. Several methods to determine marker-to-surface distance and approaches to conditioning the surface formed from the grid markers were evaluated 
for best performance. Scapular orientation obtained by palpation with the arm in positions specified by the Mallet classification was compared to estimates of scapular orientation derived by fitting the scapular template to the grid surface. Orientation was described by helical axis in the coordinate system formed by the trunk markers.

\section{Hypothesis 2}

Scapular orientations determined with palpation will be correlated with and not different from scapular orientations estimated by fitting a scapular template to the surface formed by the grid of markers. 


\section{Chapter 2}

\section{BACKGROUND AND SIGNIFICANCE}

The immediate goal of this study was to develop a clinically applicable approach to measuring scapular kinematics in individuals with minimal soft tissue overlying the scapula. The long-term goal of this research is to improve treatmentplanning strategies for patients with brachial plexus birth palsy (BPBP) and other impairments of shoulder function. Due to the difficulty of measuring scapular position and orientation, shoulder kinematics commonly describe the orientation of the humerus relative to the thorax, neglecting the important role of the scapula as an intermediary structure (Karduna et al., 2001). Abnormalities in scapular kinematics are associated with many shoulder pathologies, and improved techniques for evaluating the scapula's contribution to these pathologies can benefit the assessment and treatment strategies for these individuals (Duff et al., 2007; Illyes and Kiss, 2006; Karduna et al., 2001; Meskers et al., 1998; van Andel et al., 2009). The critical information gained is also expected to drive future work in musculoskeletal modeling, clinical trials, and basic research that will add further insight into impaired shoulder function.

Existing methods used to evaluate the scapula remain problematic and have minimal clinical applicability (Illyes and Kiss, 2006; Uhl et al., 2009). Bone pins, magnetic resonance imaging and radiographic imaging are accurate, but are either 
exceedingly invasive, expensive, or involve radiation exposure (Bey et al., 2006; Bourne et al., 2009; Graichen et al., 2000; Karduna et al., 2001; Kozin et al., 2010). Traditional skin-based methods can only measure quasi-dynamic motion; subjects pause for static measurements at various increments throughout the arm's range of motion (Meskers et al., 1998). Marker clusters mounted on the acromion process allow for dynamic motion (Illyes and Kiss, 2006; Karduna et al., 2001; van Andel et al., 2009), but have poor accuracy due to soft tissue deformation at the cluster base (Meskers et al., 2007). A moiré fringe projection technique is also being developed to determine scapular kinematics, but accuracy has not yet been demonstrated (Gomes et al., 2010).

It is imperative to accurately assess scapular function during treatment planning since variations in scapular kinematics can significantly impact intervention outcomes. For example, full range of scapular motion is required for positive outcomes following shoulder fusion procedures (Vastamaki, 1987), and contributions from the scapula lead to improvement in shoulder function following tendon transfers in BPBP patients (Kozin et al., 2010). Also, humeral osteotomies are frequently performed to improve humeral external rotation in BPBP patients, but those with scapular hypoplasia, elevation and rotation (SHEAR) deformity often exhibit poor results. The decision to undergo an alternative treatment strategy for SHEAR deformity is currently confirmed by 3D-CT, which increases the risk of future malignancy as children are especially susceptible to the detrimental effects of ionizing radiation (Kim and Newman, 2010; Nath et al., 2006). 
Clinical and surgical decision-making for BPBP children also involves functional assessments, such as the modified Mallet classification, which are used clinically to determine shoulder function and predict outcomes (Bae et al., 2003). However, these assessments only examine the ability to perform certain functional tasks and do not provide information regarding the mechanisms utilized to achieve the motions (Fitoussi et al., 2009; Mosqueda et al., 2004). Additionally, some kinematic anomalies are too subtle to be detected by clinical exam alone (Fitoussi et al., 2009). In children with BPBP, the coracoid and acromion processes develop uncharacteristically due to traction from aberrantly functioning muscles. The entire scapula is often abnormally elevated, protracted and downwardly rotated (Kambhampati et al., 2006; Nath and Paizi, 2007). In addition to atypical position and morphology, previous studies indicate that the scapula may also function differently in children with BPBP (Braman et al., 2009; Illyes and Kiss, 2006; McClure et al., 2001; Uhl et al., 2009; van Andel et al., 2009). In BPBP patients, the scapular contribution to arm elevation is greater than average (Duff et al., 2007). Increased scapular movement while reaching for high objects is likely a compensatory mechanism to overcome the neurological impairments (Kozin et al., 2010); however, current techniques do not allow elucidation of compensatory kinematic strategies. Due to these subject-specific variations in pathology, the best treatment strategy is not always readily apparent (Thatte et al., 2011).

This study used widely available motion capture technology to determine scapular kinematics during the modified Mallet classification. Motion capture is typically performed by placing markers on superficial bony landmarks to track the 
motion of a particular body segment. However, standard motion capture techniques do not work well for analyzing scapular kinematics, as the scapula moves freely underneath the skin (Bourne et al., 2009; Gomes et al., 2010; Illyes and Kiss, 2006; McClure et al., 2001; van Andel et al., 2009). The method described below takes advantage of soft tissue deformation associated with scapular orientation to extrapolate the position of the scapula beneath the overlying soft tissue. Because of the atrophy associated with palsied muscles, BPBP children tend to have less tissue covering the scapula (Hogendoorn et al., 2010) thus increasing its visibility beneath the skin. The increased visibility is often the result of scapular winging, which is an exaggerated separation between the medial border of the scapula and the thoracic wall, making BPBP patients ideal candidates for this technique. 


\section{Chapter 3}

\section{METHODS}

\section{Subjects}

Fourteen healthy, young adults were recruited; two individuals were excluded for lacking a prominent scapula. Males $(n=2)$ removed their shirts and females $(n=10)$ wore a top that exposed the region of skin covering the scapula. The right shoulders of all subjects were tested. Informed consent procedures were followed in accordance with the institution's human subjects review board.

\section{$\underline{\text { Positions }}$}

Subjects were seated on a bench with their arms held in seven positions

(Table 1). Position 1 was used to create the scapular template and positions 2-7 comprised the modified Mallet classification (Abzug et al., 2010).

Table 1 Subjects first held their arm in a neutral position to create a scapular template, then the positions of the modified Mallet classification, which are clinically relevant for assessing shoulder function.

\begin{tabular}{l|l} 
Position & Description \\
\hline 1 & Neutral position, i.e., arm relaxed at side \\
\hline 2 & Abduction in coronal plane \\
\hline 3 & Hand behind back \\
\hline 4 & External rotation at $0^{\circ}$ abduction \\
\hline 5 & Internal rotation at $0^{\circ}$ abduction
\end{tabular}




\begin{tabular}{l|l}
6 & Hand to mouth \\
\hline 7 & Hand to nape of neck
\end{tabular}

\section{Motion capture}

A 10-camera motion capture system (Motional Analysis Corporation, Santa Rosa, CA) recorded the 3D positions of retroreflective markers on the subjects' skin. For all trials, $6 \mathrm{~mm}$ spherical markers covered the landmarks on the thorax and the humerus (Table 2) at locations specified by the International Shoulder Group (ISG) (Wu et al., 2005). A 6mm spherical marker also served as a reference point on the acromion process of the scapula for all trials.

Table 2 Retroreflective, $6 \mathrm{~mm}$ spherical markers were placed on ISG recommended landmarks for the duration of data collection. *virtually reconstructed

\begin{tabular}{l|l|l} 
Thorax Markers & Humerus Markers & Scapula Marker \\
\hline C7 spinal process & Medial epicondyle & Acromion process \\
\hline T8 spinal process & Lateral epicondyle & \\
\hline Suprasternal notch & Glenohumeral joint center * & \\
\hline Xiphoid process & &
\end{tabular}

\section{Spherical fit trial}

With the markers from Table 2 applied, a spherical fit trial (Hicks and Richards, 2005) was used to approximate the glenohumeral joint center for the humerus coordinate system as recommended by the ISG (Wu et al., 2005). The trial involved moving the arm in the coronal and transverse planes, then in circumduction. 


\section{Creating the scapular template}

Subjects were seated in position 1 (Table 1) and a scapular template was created using the acromion process marker (Table 2) and 6mm 2D circular dots on the following palpated scapular landmarks: 1) acromial angle, 2) midpoint of scapular

spine, 3) trigonum spinae, 4) midpoint of medial border, and 5) inferior angle. Precise midpoints were not essential as long as they were on the scapular spine and medial border. Palpation has been previously shown to be reliable and accurate in static positions (de Groot, 1997).

\section{$\underline{\text { Validation trials }}$}

The same five marker locations used to create the scapular template were re-palpated in positions 2-7 (Table 1) to create validation trials (Fig. 1). If necessary, subjects received assistance maintaining arm position while markers were applied.

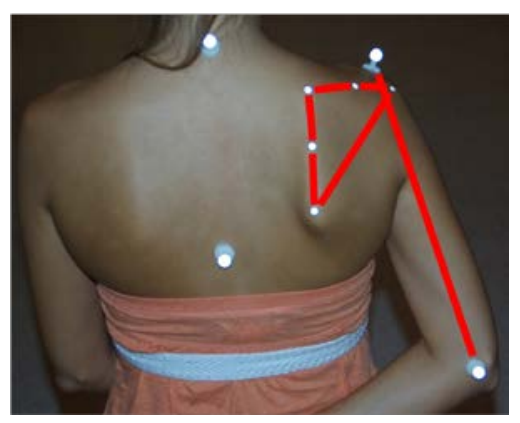

Figure 1 Validation trial of the hand behind back position with superimposed scapular and humeral orientations for demonstration purposes. 


\section{Grid surface map trials}

Once all of the validation trials were completed, the markers used to create the scapular template were removed and replaced with a grid of approximately 300 $6 \mathrm{~mm} 2 \mathrm{D}$ circular dots with $12 \mathrm{~mm}$ center-to-center spacing on strips of elastic tape (Kinesio, Albuquerque, NM, USA). The grid was placed over the skin covering the posterior region of the scapula (Fig. 2). The elastic tape conforms to the skin during movement, and the 3D positions of the markers in the grid form a surface map with the contours of the scapula evident. Subjects were instructed to achieve the same positions as in the validation trials (Table 1). For each Mallet position of the first seven subjects, three grid surface map trials were collected and the one with the humeral orientation closest to the corresponding validation trial was used. If the closest trial was not within a $15^{\circ}$ difference in humeral orientation, the position for that subject was excluded. To improve upon this, software was developed to collect data with the remaining five subjects while comparing the orientation of the humerus relative to the trunk in realtime, so that humeral orientations between methods were within a specified range. Subjects first tried to achieve within a $2^{\circ}$ difference in humeral orientation, but if this proved too difficult, these subjects were able to achieve within a $5^{\circ}$ difference. 


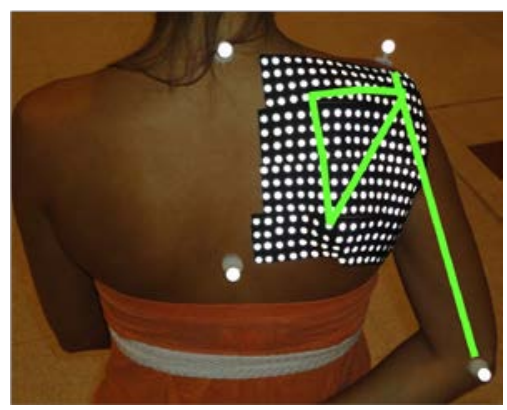

Figure 2 Surface map of the hand behind back position with superimposed scapular and humeral orientations for demonstration purposes.

\section{Surface fitting process}

All markers were rotated into the ISG recommended thorax coordinate system (Wu et al., 2005). For positions 2-7 described in Table 1, the scapular template markers were systematically rotated as a rigid body in $1^{\circ}$ increments about the three thoracic axes of the grid trial with the acromion process as the origin. The range of mathematical scapular rotation from the initial position at the center of the grid was large enough to encompass the maximum plausible movement of the scapula [ $20^{\circ}$ protraction/retraction (Y), $40^{\circ}$ medial/lateral rotation (X), $20^{\circ}$ posterior/anterior tilt

(Z)]. At each orientation of the scapular template, the absolute distances between each of the five scapular template markers and the grid surface map were summed. This was achieved by calculating the magnitude of distances relative to planes formed by applying singular value decomposition to the cluster of grid markers nearest to each scapular template marker (Fig. 3). 


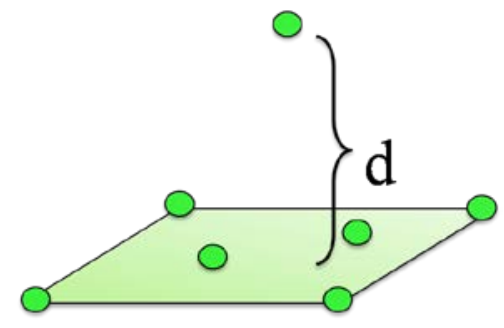

Figure 3 Visual representation of the absolute distance from one scapular landmark to its nearest plane on the grid surface formed by applying singular value decomposition.

The summed absolute differences between the five scapular template markers and the grid surface map were expected to reach a global minimum when the orientation of the scapular template matched the orientation of the underlying scapula (Fig. 4). Visual inspection demonstrated that clear global minima were always contained within the 16,000 iterations, which confirmed that the $20^{\circ} \times 40^{\circ} \times 20^{\circ}$ range was sufficient to include the actual location of the scapula. 


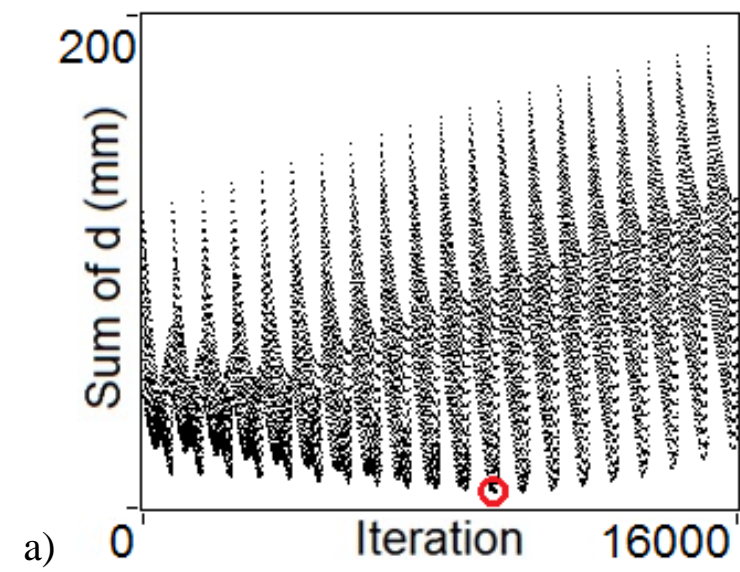

b)

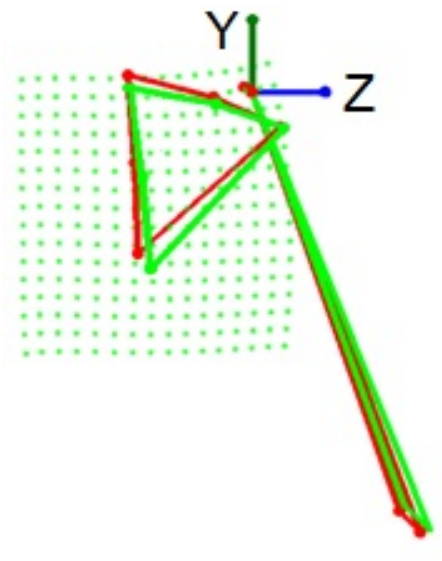

Figure 4 a) Graphical display of the surface fitting process. The global minimum error was found among 16,000 orientations tested in a $20^{\circ}$ $\times 40^{\circ} \times 20^{\circ}$ range. In this case, the sum of the absolute distances between the five scapular template markers and the surface was $6.3 \mathrm{~mm}$ at the best-fit orientation and was found at the 9,496th iteration, indicated by the circle. b) Posterior view of the surface fitting result for the hand behind back position. The scapular and humeral orientations during the validation trial are shown in red. The corresponding best-fit scapular orientation found by the surface fitting process, as well as the humeral orientation and grid during the surface map trial, are shown in green.

Different algorithms for surface fitting and mathematically manipulating the grid surface map were tested to optimize results. Specifically, three algorithms, (1) degree of filtering, (2) grid density, and (3) the number of grid markers used to create planes nearest to each scapular template marker, were varied to identify conditions that most effectively fit the scapular template to the surface. The raw data of the grid surface maps contained approximately 300 markers. The data were filtered with 3rd 
and 5th order polynomials and interpolated to contain 225, 400, 625 and 900 points. The number of grid markers used to create planes nearest to each scapular template marker was tested at 4, 5 and 6 markers. Estimates of scapular orientation derived by fitting the scapular template to the grid surface map in positions 2-7 of Table 1 were compared to the orientation of the scapula determined from the validation trial in the same position. Orientation of the scapula was described by the helical angle about each anatomical axis in the thorax coordinate system.

\section{$\underline{\text { Statistical analysis }}$}

Each Mallet position was individually tested for differences using repeated measures ANOVA (SPSS 18.0.3) with method (palpation and surface map) as an independent variable. For both scapular and humeral orientations, the helical angles between methods were compared about each anatomical axis for a total of 36 ANOVA tests. Pearson's $r$ between methods was also determined for each anatomical axis in each Mallet position for a total of 36 correlations. P-values were set to .10 and .01 for repeated measures ANOVA and Pearson's r, respectively. These p-values further strengthened the findings of this study because they are more conservative than the usual choice of .05 for a p-value. By requiring $\mathrm{p}<.10$ for ANOVA, significant differences were more likely to be found between the surface mapping and palpation methods. Since the purpose of the study was to show that the surface mapping method is equivalent to the palpation method, this was a conservative choice. Likewise, by requiring $\mathrm{p}<.01$ for regression, it was more difficult to find that results from the surface mapping method were correlated with results from palpation. Since the 
purpose of the study was to show that the results from the two methods were correlated, this was a conservative choice. Additionally, root-mean-square (RMS) errors were determined to show variability of the data and for comparison to previously published results (Karduna et al., 2001). 


\section{Chapter 4}

\section{RESULTS}

\section{Sensitivity of filtering, grid density, and cluster size}

After removing 2 of 14 subjects due to lack of a prominent scapula, 5 of 72 trials (12 subjects x 6 positions) were also removed because arm orientation exceeded a maximum acceptable difference of $15^{\circ}$ between the paired grid surface map and validation trials. The remaining 67 trials were analyzed. For all positions, the grand mean RMS errors for the helical angles between scapular orientations of the validation and surface map trials were compared using different surface fitting conditions (Fig. 5). Various conditions worked best for individual positions of each subject, but the raw data with planes determined by the six nearest surface markers produced the best overall results. 


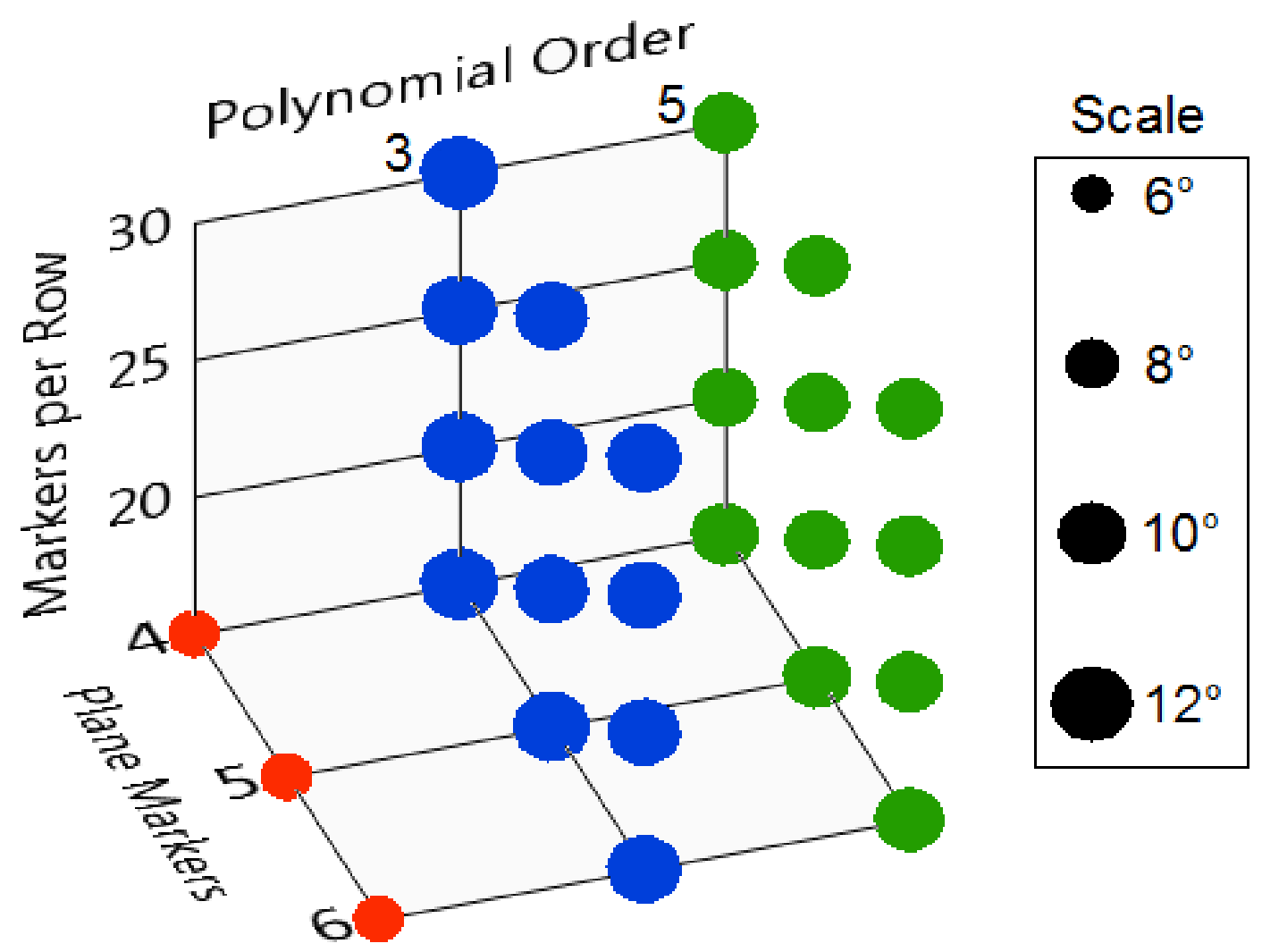

Figure 5 Effect of surface fitting conditions on the goodness of fit. The sphere diameters indicate mean RMS errors for the resultant helical angles directly between scapulae of the validation and grid surface map trials. RMS errors were calculated across all subjects for each position, and the position RMS errors were averaged. Surface fitting conditions tested were 0 th (raw), 3rd, and 5th order polynomials (red, blue, and green respectively); 15, 20, 25 and 30 interpolated markers per row (does not apply to raw data); 4, 5 and 6 nearest markers for plane determination. The smallest diameter sphere, corresponding to the best surface fitting conditions, was obtained with the raw marker data with planes based on the 6 nearest points. 


\section{Validation of surface mapping}

Using the raw data with planes determined by the six nearest surface markers, the differences between best-fit and palpated scapular orientations were calculated (Table 3). The grand mean errors in humeral orientation were within $1^{\circ}$ for each anatomical axis, meaning subjects were generally able to achieve matching arm positions between methods. Overall, the surface mapping method tends to find scapular orientations slightly more laterally rotated $\left(-1.1^{\circ}\right)$, protracted $\left(1.1^{\circ}\right)$, and anteriorly tilted $\left(-0.5^{\circ}\right)$ than actual scapular orientations. The maximum errors in scapular orientation about each anatomical axis were $3.8^{\circ}$ of lateral rotation for the hand to neck position, $3.6^{\circ}$ of protraction for the external rotation position, and $2.8^{\circ}$ of anterior tilt for the hand behind back position. 
Table 3 Surface mapping results using raw data and planes determined by the six nearest surface markers. Mean errors and RMS errors represent the difference in helical angles between methods of scapular and humeral orientations in the thorax coordinate system. Five trials were excluded because resultant humerus error was greater than $15^{\circ}$. Significant differences in mean error were determined by repeated measures ANOVA for each anatomical axis of each position, with method (palpation and surface map) as an independent variable.

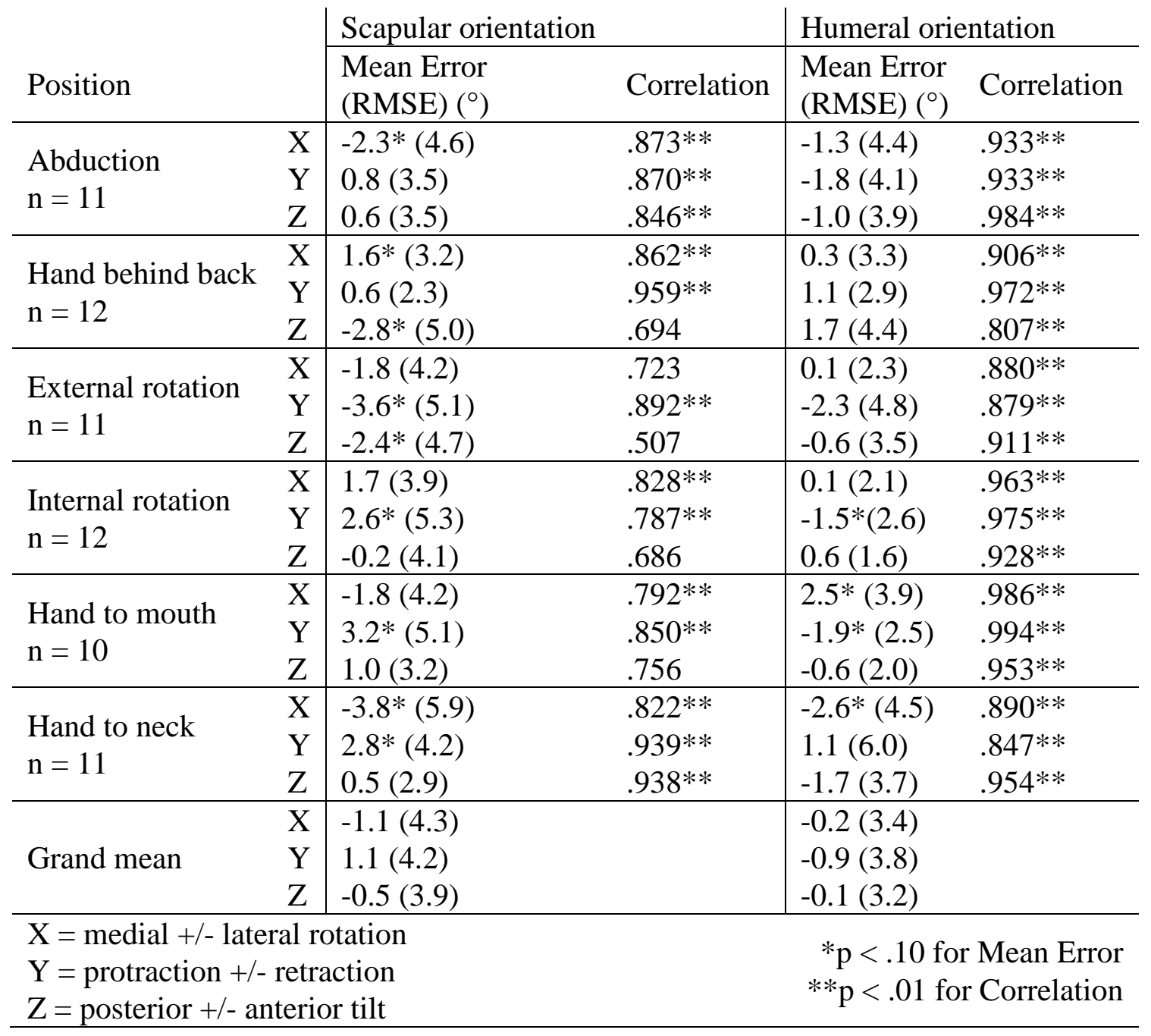




\section{Chapter 5}

\section{DISCUSSION}

\section{Comparison to existing methods}

The largest absolute mean error for the surface mapping method was $3.8^{\circ}$ with an RMS error of $5.9^{\circ}$ in lateral rotation during the hand to nape of neck position of the modified Mallet classification. These results compare favorably with three existing studies (Karduna et al., 2001; Meskers et al., 2007; van Andel et al., 2009). Karduna et al. (2001) independently validated both an acromial method and a scapula tracker method to bone pins. Maximal RMS errors were $10^{\circ}$ in medial/lateral rotation during horizontal adduction for the tracker method and $11.4^{\circ}$ in protraction/retraction during abduction for the acromial method. Meskers et al. (2007) compared a tripod method to a skin-fixed recording method similar to the acromial method and scapula tracker method of Karduna et al. (2001), respectively. Maximal mean errors were $7^{\circ}$ during forward flexion and $13^{\circ}$ during abduction, both in medial/lateral rotation. van Andel et al. (2009) compared an acromion marker cluster to a scapula locator similar to the scapula tracker method of Karduna et al. (2001). Maximal mean errors were $6^{\circ}$ for forward flexion and abduction and $8.4^{\circ}$ in protraction/retraction during internal/external rotation at $90^{\circ}$ abduction. 


\section{$\underline{\text { Sources of error }}$}

There are a few possible sources of error that can account for the differences in scapular orientation determined by palpation in the validation trials versus surface mapping. The elastic tape used to create the surface maps conformed to the skin very well in tension, but occasionally rippled under excessive compression near the acromial angle when the arm was elevated. However, these errors were mitigated because the rippling only affected the 6-point plane formed in the region of the acromial angle marker and thus the distance to it, which was summed with the four other scapular landmark distances. Other sources of error include or can be attributed to less than perfect matching of arm positions between the validation and corresponding surface mapping trials. This could be due to different spinal postures or shoulder shrugging that would cause the scapula to be in a slightly different orientation. Although some of the differences between surface mapping and validation trials were statistically significant, the errors are small enough to be clinically insignificant.

\section{Concluding remarks}

This approach is an improved method for noninvasive determination of scapular kinematics, and will provide the data necessary to gain knowledge regarding the scapula's contribution to shoulder motion and the neuromechanics of shoulder pathologies. In addition to its research implications, this approach has potential for use in clinical medicine. Many clinics already have access to motion capture technology for gait analysis and can take advantage of the non-invasive nature of the surface mapping method for upper extremity analysis. Further refinement of this approach is 
expected to provide physicians with the ability to obtain previously elusive information concerning scapular kinematics that can facilitate the formation of novel, patient-specific treatment plans directed at improving shoulder function. 


\section{REFERENCES}

Abzug, J.M., Chafetz, R.S., Gaughan, J.P., Ashworth, S., Kozin, S.H., 2010. Shoulder function after medial approach and derotational humeral osteotomy in patients with brachial plexus birth palsy. Journal of Pediatric Orthopaedics. 30 (5), 469-474.

Bae, D.S., Waters, P.M., Zurakowski, D., 2003. Reliability of three classification systems measuring active motion in brachial plexus birth palsy. Journal of Bone and Joint Surgery (American). 85-A (9), 1733-1738.

Bey, M.J., Zauel, R., Brock, S.K., Tashman, S., 2006. Validation of a new model-based tracking technique for measuring three-dimensional, in vivo glenohumeral joint kinematics. Journal of Biomechanical Engineering. 128 (4), 604609.

Bourne, D.A., Choo, A.M., Regan, W.D., Macintyre, D.L., Oxland, T.R., 2009. A new subject-specific skin correction factor for three-dimensional kinematic analysis of the scapula. Journal of Biomechanical Engineering. 131 (12), 121009.

Braman, J.P., Engel, S.C., Laprade, R.F., Ludewig, P.M., 2009. In vivo assessment of scapulohumeral rhythm during unconstrained overhead reaching in asymptomatic subjects. Journal of Shoulder and Elbow Surgery. 18 (6), 960-967.

de Groot, J.H., 1997. The variability of shoulder motions recorded by means of palpation. Clinical Biomechanics. 12 (7-8), 461-472. 
Duff, S.V., Dayanidhi, S., Kozin, S.H., 2007. Asymmetrical shoulder kinematics in children with brachial plexus birth palsy. Clinical Biomechanics. 22 (6), 630-638.

Fitoussi, F., Maurel, N., Diop, A., Laassel, E.M., Ilharreborde, B., Presedo, A., Mazda, K., Pennecot, G.F., 2009. Upper extremity kinematics analysis in obstetrical brachial plexus palsy. Orthopaedics \& Traumatology: Surgery \& Research. 95 (5), 336-342.

Gomes, P.F., Sesselmann, M., Faria, C.D., Araujo, P.A., Teixeira-Salmela, L.F., 2010. Measurement of scapular kinematics with the moire fringe projection technique. Journal of Biomechanical Engineering. 43 (6), 1215-1219.

Graichen, H., Stammberger, T., Bonel, H., Haubner, M., Englmeier, K.H., Reiser, M., Eckstein, F., 2000. Magnetic resonance-based motion analysis of the shoulder during elevation. Clinical Orthopaedics and Related Research (370), 154163.

Hicks, J.L., Richards, J.G., 2005. Clinical applicability of using spherical fitting to find hip joint centers. Gait \& Posture. 22 (2), 138-145.

Hogendoorn, S., van Overvest, K.L., Watt, I., Duijsens, A.H., Nelissen, R.G., 2010. Structural changes in muscle and glenohumeral joint deformity in neonatal brachial plexus palsy. Journal of Bone and Joint Surgery (American). 92 (4), 935-942.

Illyes, A., Kiss, R.M., 2006. Method for determining the spatial position of the shoulder with ultrasound-based motion analyzer. Journal of Electromyography and Kinesiology. 16 (1), 79-88. 
Kambhampati, S.B., Birch, R., Cobiella, C., Chen, L., 2006. Posterior subluxation and dislocation of the shoulder in obstetric brachial plexus palsy. Journal of Bone and Joint Surgery (British). 88 (2), 213-219.

Karduna, A.R., McClure, P.W., Michener, L.A., Sennett, B., 2001. Dynamic measurements of three-dimensional scapular kinematics: A validation study. Journal of Biomechanical Engineering. 123 (2), 184-190.

Kim, J.E., Newman, B., 2010. Evaluation of a radiation dose reduction strategy for pediatric chest ct. American Journal of Roentgenology. 194 (5), 11881193.

Kozin, S.H., Chafetz, R.S., Shaffer, A., Soldado, F., Filipone, L., 2010. Magnetic resonance imaging and clinical findings before and after tendon transfers about the shoulder in children with residual brachial plexus birth palsy: A 3-year follow-up study. Journal of Pediatric Orthopaedics. 30 (2), 154-160.

McClure, P.W., Michener, L.A., Sennett, B.J., Karduna, A.R., 2001. Direct 3-dimensional measurement of scapular kinematics during dynamic movements in vivo. Journal of Shoulder and Elbow Surgery. 10 (3), 269-277.

Meskers, C.G., van de Sande, M.A., de Groot, J.H., 2007. Comparison between tripod and skin-fixed recording of scapular motion. Journal of Biomechanics. 40 (4), 941-946.

Meskers, C.G., Vermeulen, H.M., de Groot, J.H., van Der Helm, F.C., Rozing, P.M., 1998. 3d shoulder position measurements using a six-degree-of-freedom electromagnetic tracking device. Clinical Biomechanics. 13 (4-5), 280-292. 
Mosqueda, T., James, M.A., Petuskey, K., Bagley, A., Abdala, E., Rab, G., 2004. Kinematic assessment of the upper extremity in brachial plexus birth palsy. Journal of Pediatric Orthopaedics. 24 (6), 695-699.

Nath, R.K., Melcher, S.E., Paizi, M., 2006. Surgical correction of unsuccessful derotational humeral osteotomy in obstetric brachial plexus palsy: Evidence of the significance of scapular deformity in the pathophysiology of the medial rotation contracture. Journal of Brachial Plexus and Peripheral Nerve Injury. 1 (9), 1-7.

Nath, R.K., Paizi, M., 2007. Scapular deformity in obstetric brachial plexus palsy: A new finding. Surgical and Radiologic Anatomy. 29 (2), 133-140.

Thatte, M.R., Agashe, M.V., Rao, A., Rathod, C.M., Mehta, R., 2011. Clinical outcome of shoulder muscle transfer for shoulder deformities in obstetric brachial plexus palsy: A study of 150 cases. Indian Journal of Plastic Surgery. 44 (1), 21-28.

Uhl, T.L., Kibler, W.B., Gecewich, B., Tripp, B.L., 2009. Evaluation of clinical assessment methods for scapular dyskinesis. Arthroscopy: The Journal of Arthroscopic and Related Surgery. 25 (11), 1240-1248.

van Andel, C., van Hutten, K., Eversdijk, M., Veeger, D., Harlaar, J., 2009. Recording scapular motion using an acromion marker cluster. Gait \& Posture. 29 (1), 123-128.

Vastamaki, M., 1987. Shoulder arthrodesis for paralysis and arthrosis. Acta Orthopaedica Scandinavia. 58 (5), 549-553. 
Wu, G., van der Helm, F.C.T., Veeger, H.E.J., Makhsous, M., Van Roy, P., Anglin, C., Nagels, J., Karduna, A.R., McQuade, K., Wang, X.G., Werner, F.W., Buchholz, B., 2005. Isb recommendation on definitions of joint coordinate systems of various joints for the reporting of human joint motion - part ii: Shoulder, elbow, wrist and hand. Journal of Biomechanics. 38 (5), 981-992. 


\section{Appendix A}

\section{ADDITIONAL COMMENTS}

1. For both of our hypotheses, we chose ANOVA paired with Pearson's r instead of intraclass correlation coefficients (ICC) because we believe that ICC lacks the sensitivity to provide meaningful results about how comparable surface mapping is to palpation.

2. It may seem that the methodology forces subjects to have matching arm positions between the two measures for each Mallet posture, precluding a fair analysis of specific aim 1 . This is necessary because we are interested in validating the computational algorithm of specific aim 2 and in order to do so, specific aim 1 is an assumption that must be met.

3. The software developed to provide real-time feedback of humeral orientation extracts coordinate data from the dynamic link library (DLL) of Cortex motion capture software (Motional Analysis Corporation, Santa Rosa, CA). Then in the thoracic coordinate system, it calculates the difference of the resultant helical angle between the humeral coordinate systems of the previously collected validation trial and the real-time grid surface map trial. When the helical angle is within the limit $\left(2^{\circ}\right.$ or $\left.5^{\circ}\right)$, the software saves that frame of coordinate data. 
4. Results from the surface mapping method compared favorably to existing acromial cluster and scapula locator methods. The acromial cluster method determines orientation from three sensors in close proximity to each other, so errors from soft tissue deformation at the base become amplified. The scapula tracker method must be manually manipulated to stay in contact at the correct points on the scapula, which leaves ample room for human error.

For the surface mapping method, we believe that the raw data worked best because the grid density was sufficient without being interpolated to increase the density at the expense of being filtered and smoothed. To prevent the possibility of using collinear points, clusters of at least four grid markers were needed to determine surface planes closest to each scapular landmark. We discovered that using less than six surface markers seemed to lack robustness and more than six surface markers were not necessarily close to each scapular landmark. 
Appendix B

IRB APPROVAL LETTER 


\section{ఏ}

Research Office
210 Hullihen Hall

University of Delaware

Newark, Delaware 19716-1551

Ph: 302/831-2136

Fax: 302/831-2828
DATE:

TO:

FROM:

STUDY TITLE:

IRB REFERENCE \#:

SUBMISSION TYPE:

ACTION:

APPROVAL DATE:

EXPIRATION DATE:

REVIEW TYPE:

REVIEW CATEGORY:
August 11, 2010

James Richards

University of Delaware IRB

[183542-1] Identification of scapular position using surface mapping

New Project

APPROVED

August 11, 2010

August 10, 2011

Expedited Review

Expedited review category \# 7

Thank you for your submission of New Project materials for this research study. The University of Delaware IRB has APPROVED your submission. This approval is based on an appropriate risk/benefit ratio and a study design wherein the risks have been minimized. All research must be conducted in accordance with this approved submission.

This submission has received Expedited Review based on the applicable federal regulation.

Please remember that informed consent is a process beginning with a description of the study and insurance of participant understanding followed by a signed consent form. Informed consent must continue throughout the study via a dialogue between the researcher and research participant. Federal regulations require each participant receive a copy of the signed consent document.

Please note that any revision to previously approved materials must be approved by this office prior to initiation. Please use the appropriate revision forms for this procedure.

All SERIOUS and UNEXPECTED adverse events must be reported to this office. Please use the appropriate adverse event forms for this procedure. All sponsor reporting requirements should also be followed.

Please report all NON-COMPLIANCE issues or COMPLAINTS regarding this study to this office.

Please note that all research records must be retained for a minimum of three years.

Based on the risks, this project requires Continuing Review by this office on an annual basis. Please use the appropriate renewal forms for this procedure. 
If you have any questions, please contact Elizabeth Peloso at 302-831-8619 or epeloso@udel.edu. Please include your study title and reference number in all correspondence with this office. 